\title{
Assessing iron overload: are we there yet?
}

\author{
Paul C. Kruger ${ }^{1}$, Michael F. Leahy ${ }^{1,2}$ and John K. Olynyk $k^{2,3,4,5}$
}

Iron overload occurs in many haematologic disorders and causes significant morbidity. The advantages of magnetic resonance imaging in quantifying liver iron concentration continue to mount and the association between iron overload and increased mortality post allogeneic stem cell transplant needs further attention.

In this issue of Clinical Cancer Research, Wermke et al ${ }^{1}$ publish results of an observational study of magnetic-resonance imaging (MRI) assessment of liver iron content (LIC) in 88 patients undergoing allogeneic stem cell transplantation (allo-SCT) for either myelodysplastic syndrome (MDS) $(n=24)$ or acute myeloid leukaemia (AML) $(n=64)$. Systemic iron overload (SIO) in these conditions is a consequence of ineffective erythropoiesis and blood transfusions used to treat anaemia due to either the disease itself or cytotoxic chemotherapy. It is a frequent and significant problem in these conditions and may result in organ damage due to iron deposition in the liver, heart, pancreas, and endocrine glands. ${ }^{2}$ Furthermore, SIO results in increased risks of serious infections. Thus, assessment of body iron stores and quantitation of LIC is a relevant part of the clinical assessment of such patients. ${ }^{3}$

The authors measured the LIC using MRI and assessed the relationships between pretransplant LIC, serum iron biochemistry parameters (transferrin saturation (TRS) and serum ferritin), and post allo-SCT outcomes. Eighty-five of 88 patients had serum ferritin levels elevated to $>400 \mathrm{ng} / \mathrm{mL}$, whilst $44 \%$ had serum TRS values $>80 \%$. The median LIC was $125 \mathrm{umol} / \mathrm{g}$ (ULN: $36 \mathrm{umol} / \mathrm{g}$; range $25-$ $350 \mathrm{umol} / \mathrm{g}$ ) prior to allo-SCT. Positive correlations were observed between LIC and transfusion history $(r=0.67 ; p<0.001)$ and serum ferritin $(r=0.640 ; p<0.001)$.

The most widely available tools to assess iron overload are the indirect markers serum ferritin and TRS. However, they are sensitive but not specific as ferritin is also an acute phase reactant whilst TRS has a day to day variability. Indeed in the current study, a ferritin value $>1000 \mathrm{ng} / \mathrm{ml}$ provided a specificity of only $32 \%$ for detection of LIC > $125 \mathrm{umol} / \mathrm{g}$. More accurate quantitation of LIC is possible via non-invasive methods, such as $\mathrm{MRI}$, or invasive approaches, such as liver biopsy. Liver biopsy is rarely clinically appropriate in patients with MDS and AML who are often thrombocytopaenic. Liver MRI has been clearly shown to accurately assess LIC over a large range of iron concentrations, ${ }^{4,5}$ either by signal intensity ratio or relaxometry methods. ${ }^{6}$ Of all the reported MRI methods, FerriScan ${ }^{\circledR}$ has been licenced by regulatory authorities in Europe, USA and Australia. ${ }^{7}$ (INSERT FIGURE AT THIS POINT)

Labile plasma iron (LPI), a toxic redox active component of non-transferrin-bound iron and a major mediator of tissue damage, was also analysed in this study as a diagnostic tool. Nine patients tested positive for LPI with a median of $0.95 \mathrm{LPI}$ units (range:0.71-3.21 LPI units), although there was no significant correlation between pre-allo-SCT LPI 
concentrations and LIC, transfusion burden, or ferritin. Nor was there any correlation between enhanced LPI and these markers in over half $(13 / 24)$ of the screened patients. The role of LPI as a diagnostic tool remains investigational..$^{8}$ Nevertheless, LPI remains an important contributor to iron toxicity as levels rise after chemotherapy in patients with AML. Further, it offers a potential target for therapy as it is directly chelatable. Further clarification of the role of iron chelation in these conditions is required. Vigorous iron chelation in the pre-transplantation period may be important, but the benefits of chelation posttransplantation are uncertain. ${ }^{2}$

Wermke and colleagues clearly show the importance of iron status for the prediction of outcome of allo-SCT. Patients with high LIC had an increased cumulative incidence of non-relapse mortality (at day 100 , $<125 \mathrm{umol} / \mathrm{g}$ vs. $\geq 125 \mathrm{umol} / \mathrm{g}$ : $4.7 \%$ vs. $27.3 \%$, $\mathrm{p}=0.028) .{ }^{1}$ Other studies have also documented the association between iron overload and decreased survival post allo-SCT in both malignant and non-malignant haematological conditions. ${ }^{9,10}$ Although serum ferritin has been used as a marker in these studies instead of MRI assessment of LIC, it still adds to the evidence base that iron overload is frequent and deleterious in this population of patients. Reducing the number of red blood cell (RBC) transfusions may reduce non-relapse mortality. A Swiss study in patients receiving intensive chemotherapy for haematological malignancy and stem cell transplantation demonstrated that a change from a double to single unit RBC transfusion policy is safe, sufficient to treat anaemia, and associated with a reduction by $25 \%$ in RBC transfusion requirements and reduction in other complications associated with RBC transfusion. ${ }^{11}$ There was no evidence of more severe bleeding or more platelet transfusions during the single-unit period and the overall survival was similar in both single and double unit cohorts. Clearly, further studies to assess the impact of reduced RBC transfusion support in intensive chemotherapy and stem cell transplantation are indicated.

The work of Wermke et al adds to the growing body of literature in renal ${ }^{12}$ and hepatological ${ }^{5}$ fields which documents the non-specificity of serum ferritin as a marker of iron overload and the superiority of MRI based methods for documentation of LIC. ${ }^{4,5,12}$ Whilst the evidence is clear, the major limitations of such non-invasive approaches relate to availability, standardisation and cost. The MRI approach described in the current study (1.5 Tesla scanner, protocol based on gradient recalled echo sequences) is not licenced by regulatory authorities and does not have internationally standardised quality controls. Whilst FerriScan ${ }^{\circledR}$ is standardised and does have regulatory approval and quality control systems, it is not reimbursable in many countries, limiting availability. However, it may be possible to mount arguments for reimbursement based on studies such as those of Wermke et al which indicate that early identification of at-risk patients and early treatment with iron chelation therapy might reduce iron-mediated toxicity and potentially improve outcomes. This seems innately sensible given the large resource costs of allo-SCT.

Wermke and colleagues have provided compelling information on the dangers of SIO post allo-SCT which adds to our current understanding of iron overload increasing morbidity and mortality and decreasing quality of life. Further, knowledge of the dangers of SIO has ramifications for patients with non-malignant haematological conditions. It is now time to revise the standard of care for diagnosis of SIO to include liver MRI as the gold standard in assessing LIC. Such technologies will allow definitive evaluation of the impact of early chelation treatment to reduce iron burden and ensure that patients are in optimal condition prior to, or following, allo-SCT so that transplantation outcomes can continue to improve. 


\section{References}

1. Wermke M, Schmidt A, Middeke JM, Sockel $\mathrm{K}$, von Bonin M, Schonefeldt C, et al. MRIbased liver iron content predicts for nonrelapse mortality in MDS and AML patients undergoing allogeneic stem cell transplantation. Clinical Cancer Research 2012.

2. de Witte T. The role of iron in patients after bone marrow transplantation. Blood Reviews 2008;22 Suppl. 2: S22-S28.

3. Virtanen JM, Remes KJ, Itala-Remes MA, Saunavaara JP, Komu ME, Partanen AM, et al. The relationship between cardiac and liver iron evaluated by MR imaging in haematological malignancies and chronic liver disease. Blood Cancer Journal 2012;2:e49.

4. Gandon Y, Olivie D, Guyader D, Aube C, Oberti F, Sebille V, et al. Non-invasive assessment of hepatic iron stores by MRI. Lancet 2004;363:357-362.

5. St Pierre TG, Clark PR, Chua-anusorn W, Fleming AJ, Jeffrey GP, Olynyk JK, et al. Noninvasive measurement and imaging of liver iron concentrations using proton magnetic resonance. Blood 2005;105:855861.

6. Echeverria JMA, Castiella A, Emparanza JI. Quantification of iron concentration in the liver by MRI. Insights Imaging 2012;3:173-180.

7. Olynyk JK, Gan E, Tan T. Predicting iron overload in hyperferritinemia. Clinical
Gastroenterology and Hepatology 2009;7:359-362.

8. Bird RJ, Kenealy M, Forsyth C, Wellwood J, Leahy MF, Seymour JF, et al. When should iron chelation therapy be considered in patients with myelodysplasia and other bone marrow failure syndromes with iron overload? Internal Medicine Journal 2012;42:450-455.

9. Bazuave GN, Buser A, Gerull S, Tichelli A, Stern M. Prognostic impact of iron parameters in patients undergoing allo-SCT. Bone Marrow Transplantation 2012;47:60-64.

10. Armand P, Kim HT, Cutler CS, Ho VT, Koreth J, Alyea EP, et al. Prognostic impact of elevated pretransplantation serum ferritin in patients undergoing myeloablative stem cell transplantation. Blood 2007;109:4586-4588.

11. Berger MD, Gerber B, Arn K, Senn O, Schanz $U$, Stussi G. Significant reduction of red blood cell transfusion requirements by changing from a double-unit to a single-unit transfusion policy in patients receiving intensive chemotherapy or stem cell transplantation. Haematologica 2012;97:116122.

12. Ferrari P, Kulkarni H, Dheda S, Betti S, Harrison C, St Pierre TG, et al. Serum iron markers are inadequate for guiding iron repletion in chronic kidney disease. Clinical Journal of the American Society of Nephrology 2011;6:77-83.

\section{Original figure}

Already submitted separately.

\section{Authors' affiliations}

${ }^{1}$ Department of Haematology, Fremantle Hospital, Fremantle, Western Australia; ${ }^{2}$ School of Medicine and Pharmacology, University of Western Australia, ${ }^{3}$ Department of Gastroenterology, Fremantle Hospital, Fremantle, Western Australia; ${ }^{4}$ Curtin Health Innovation Research Institute, 
Curtin University, Bentley, Western Australia; ${ }^{5}$ Institute for Immunology \& Infectious Diseases, Murdoch University, Murdoch, Western Australia.

\section{Corresponding author}

John K. Olynyk, Department of Gastroenterology, Fremantle Hospital, PO Box 480, Fremantle 6959, Western Australia. Phone: +618 94312480; Fax: +618 94312340; E-mail:

john.olynyk@health.wa.gov.au

\section{Disclosure of Potential Conflicts of Interest \\ None identified.}

\section{Authors' Contributions}

All authors contributed equally to the manuscript.

\section{Running title}

Iron overload and non-relapse mortality post stem cell transplant

\section{Figure legend}

Advantages and disadvantages of different modalities to assess iron overload: MDS myelodysplastic syndrome; AML - acute myeloid leukaemia; MRI - magnetic resonance imaging; LIC - liver iron content; TRS - transferrin saturation; ref: St Pierre TG, Clark PR, Chua-anusorn W, Fleming AJ, Jeffrey GP, Olynyk JK, et al. Noninvasive measurement and imaging of liver iron concentrations using proton magnetic resonance. Blood 2005;105:855-861. 